\title{
Current and future pharmacological therapies for NAFLD/NASH
}

\author{
Yoshio Sumida $^{1} \cdot$ Masashi Yoneda $^{1}$
}

Received: 27 September 2017 / Accepted: 17 November 2017/Published online: 16 December 2017

(C) The Author(s) 2017. This article is an open access publication

\begin{abstract}
Nonalcoholic fatty liver disease (NAFLD) is the most prevalent liver disease worldwide, and there is no approved pharmacotherapy. The efficacy of vitamin E and pioglitazone has been established in nonalcoholic steatohepatitis (NASH), a progressive form of NAFLD. GLP1RA and SGLT2 inhibitors, which are currently approved for use in diabetes, have shown early efficacy in NASH, and also have beneficial cardiovascular or renal effects. Innovative NASH therapies include four main pathways. The first approach is targeting hepatic fat accumulation. Medications in this approach include modulation of peroxisome proliferator-activator receptors (e.g., pemafibrate, elafibranor), medications targeting farnesoid $\mathrm{X}$ receptor axis [obeticholic acid; OCA)], inhibitors of de novo lipogenesis (aramchol, ACC inhibitor), and fibroblast growth factor-21 analogues. A second target is oxidative stress, inflammation, and apoptosis. This class of drug includes apoptosis signaling kinase 1 (ASK1) inhibitor and emricasan (an irreversible caspase inhibitor). A third target is intestinal microbiomes and metabolic endotoxemia. Several agents are in ongoing trials, including IMMe124, TLR4 antagonist, and solithromycin (macrolide antibiotics). The final target is hepatic fibrosis, which is strongly associated with all-cause or liver-related mortality in NASH. Antifibrotic agents are a cysteine-cysteine motif chemokine receptor-2/5 antagonist (cenicriviroc; CVC) and galectin 3 antagonist. Among a variety of medications in development, four agents such as OCA, elafibranor, ASK1
\end{abstract}

Yoshio Sumida

sumida.yoshio.500@mail.aichi-med-u.ac.jp

1 Division of Hepatology and Pancreatology, Department of Internal Medicine, Aichi Medical University, Nagakute, Aichi 480-1195, Japan inhibitor, and CVC are currently being evaluated in an international phase 3 trial for the treatment of NASH. Within the next few years, the availability of therapeutic options for NASH will hopefully curb the rising trend of NASH-related diseases.

Keywords Diabetes · Hepatic fibrosis - NASH - SGLT2 inhibitor - GLP-1 receptor agonist

\section{Introduction}

Nonalcoholic fatty liver disease (NAFLD) is the most prevalent chronic liver disease. One-fourth of the adult population is now suffering from NAFLD worldwide $[1,2]$. Nonalcoholic steatohepatitis (NASH), the aggressive form of NAFLD, can progress to cirrhosis and hepatocellular cancer (HCC) and is rapidly becoming the leading cause for end-stage liver disease or liver transplantation [3]. In Japan, liver-related diseases, such as cirrhosis and HCC, are now the third leading cause of death in type 2 diabetes mellitus (T2DM) [4], which is closely associated with NAFLD. It is estimated that the prevalence of diagnosed NASH will reach 18 million by 2027 in US, Japan, and EU 5 (England, France, Germany, Italy, and Spain). Lifestyle interventions, such as dietary caloric restriction and exercise, currently the cornerstone of therapy for NASH/NAFLD, can be difficult to achieve and maintain, underscoring the dire need for pharmacotherapy. However, there are no approved pharmacotherapies for NASH/ NAFLD. This review presents the agents currently used in managing NASH/NAFLD and their pharmacologic targets. It also provides an overview of NAFLD agents currently under development. 


\section{Currently recommended pharmacotherapies in the practice guidelines from US, Europe, and Japan}

Evidence-based practice guidelines for the management of NASH/NAFLD were published by the American Association for the Study of Liver Disease (AASLD) in 2012 [5], the Japan Society of Gastroenterology (JSG)-Japan Society of Hepatology (JSH) in 2014 [6], and the European Association for the Study of the Liver (EASD)-European Association for the Study of Diabetes (EASL)-European Association for the Study of Obesity (EASO) in 2016 [7]. The AASLD recently proposed NAFLD "guidance" to help clinicians understand and implement the most recent evidence [8]. In summary, pioglitazone and vitamin $\mathrm{E}$ are now recommended as pharmacotherapies for biopsy-proven NASH patients with and without diabetes, respectively, although long-term efficacy or safety should be established (Table 1) [5-8].

\section{Whom to treat}

Dietary changes and lifestyle modifications are now the first-line therapy for patients with NASH. Body weight reduction cannot be achieved in a lot of patients. The most difficult question to answer is what will be the target population of NAFLD/NASH pharmacologic treatment. According to the practice guideline from Europe proposed in 2016, pharmacotherapies should be considered for NASH patients with fibrosis stage 2 or higher and with early stage fibrosis with high risk of fibrosis progression (older age, diabetes, metabolic syndrome, increased ALT, and high necroinflammatory activity) [5]. According to a meta-analysis evaluating five adult NAFLD cohort studies, the presence of advanced fibrosis (stage 2 or more) is the most important predictor of liver-related mortality in NAFLD patients [9]. The AASLD guidance also suggests that pharmacotherapies aimed primarily at improving liver disease should be limited to patients with NASH and fibrosis [8]. Therefore, patients with non-aggressive type of NAFLD (NAFL or NASH stage 0) does not require liverspecific treatments, although the prevention of

Table 1 The summary of recommended pharmacotherapies for NASH/NAFLD in guidelines or guidance

\begin{tabular}{|c|c|c|c|c|}
\hline & AGA/AALSLD (2012) & JSG/JSH (2014) & EASL/EASD/EAO (2016) & AASLD guidance (2017) \\
\hline Vitamin E & $\begin{array}{l}\text { First-line therapy for biopsy- } \\
\text { proven NASH without diabetes } \\
\text { and cirrhosis }(800 \mathrm{mg} / \text { day })\end{array}$ & Recommended & $\begin{array}{l}\text { Not firmly recommended, } \\
\text { but could be used }\end{array}$ & $\begin{array}{l}\text { May be considered in biopsy- } \\
\text { proven NASH without diabetes } \\
\text { and cirrhosis ( } 800 \mathrm{mg} / \text { day) } \\
\text { Discuss benefit and risk with } \\
\text { patients }\end{array}$ \\
\hline UDCA & Not recommended & Not recommended & Not mentioned in detail & Not recommended \\
\hline Pioglitazone & $\begin{array}{l}\text { Can be used in patients with } \\
\text { biopsy-proven NASH }\end{array}$ & $\begin{array}{l}\text { Recommended in } \\
\text { NASH with insulin } \\
\text { resistance }\end{array}$ & $\begin{array}{l}\text { Not firmly recommended, } \\
\text { but could be used }\end{array}$ & $\begin{array}{l}\text { Can be used in patients with } \\
\text { biopsy-proven NASH } \\
\text { Discuss benefit and risk with } \\
\text { patients }\end{array}$ \\
\hline Metformin & $\begin{array}{l}\text { Not recommended as a specific } \\
\text { treatment for NASH }\end{array}$ & $\begin{array}{l}\text { Not recommended as a } \\
\text { specific treatment for } \\
\text { NASH }\end{array}$ & Insufficient evidence & $\begin{array}{l}\text { Not recommended as a specific } \\
\text { treatment for NASH }\end{array}$ \\
\hline GLP-1RA & Not mentioned & Not mentioned & Not mentioned & $\begin{array}{l}\text { Premature as a specific treatment } \\
\text { for NASH }\end{array}$ \\
\hline$\omega 3$ fatty acid & $\begin{array}{c}\text { May be considered in NAFLD } \\
\text { with hypertriglyceridemia }\end{array}$ & Not mentioned & $\begin{array}{l}\text { Reduced lipid in plasma } \\
\text { and liver, but no } \\
\text { evidence related to } \\
\text { NASH }\end{array}$ & $\begin{array}{l}\text { Not recommended as a specific } \\
\text { treatment for NASH } \\
\text { May be considered in NAFLD } \\
\text { with hypertriglyceridemia }\end{array}$ \\
\hline Statin & Can be used to treat dyslipidemia & $\begin{array}{l}\text { Recommended for } \\
\text { hypercholesterolemia }\end{array}$ & $\begin{array}{l}\text { Can be used to reduce } \\
\text { LDL-C and prevent } \\
\text { cardiovascular risk }\end{array}$ & $\begin{array}{l}\text { Can be used to treat dyslipidemia } \\
\text { Should be avoided in } \\
\text { decompensated cirrhosis }\end{array}$ \\
\hline Pentoxifylline & Not mentioned & $\begin{array}{l}\text { Recommended, but } \\
\text { commercially } \\
\text { unavailable in Japan }\end{array}$ & Not mentioned & Not mentioned \\
\hline OCA & Not mentioned & Not mentioned & Not mentioned & $\begin{array}{l}\text { Off-label use not recommended } \\
\text { (approved for PBC in USA) }\end{array}$ \\
\hline
\end{tabular}


cardiovascular or renal diseases are strategically essential in them (Fig. 1).

\section{How to treat (Fig. 2)}

\section{Antioxidants/hepatoprotective drugs}

Vitamin E Oxidative stress has been implicated to have an important role in the progression of NASH [10, 11]. Vitamin $\mathrm{E}$ is well known as a free radical scavenger, and has been expected for the treatment of NASH. We previously reported that vitamin $\mathrm{E}$ treatment for 1 year reduced serum transaminase activities as well as transforming growth factor-beta1 in adult NASH patients who were refractory to dietary intervention $[12,13]$. In pioglitazone versus vitamin $\mathrm{E}$ versus Placebo for the Treatment of Nondiabetic Patients with Nonalcoholic Steatohepatitis (PIVENS) trial, vitamin E (800 mg/day) is superior to placebo for the improvements of NASH histology in adults NASH without diabetes and cirrhosis [14]. According to a random-effects model analysis of the five studies, vitamin E significantly reduced serum hepatobiliary enzymes, hepatic steatosis, inflammation, and hepatocellular ballooning compared with the control group [15]. In those studies, however, fibrosis improvement was not confirmed. In Japan, long-term vitamin E treatments (300 mg/day) for more than 2 years can ameliorate hepatic fibrosis in NASH patients, especially in those whose serum transaminase activities and insulin resistance can be improved [16]. This result has suggested that metabolic factors should be controlled even when vitamin $\mathrm{E}$ is administrated. Although vitamin $\mathrm{E}$ is now recommended only for biopsy-proven NASH patients without diabetes on the basis of PIVENS trial, it is associated with histological improvement regardless of diabetic status [17]. However, the primary concern regarding vitamin E for NASH treatment has been the potential for toxicity with long-term or high-dose use. Vitamin E treatment may increase all-cause mortality [18], prostatic cancer (SELECT trial) [19], and hemorrhagic stroke [20], although several conflicting results exist. When vitamin $\mathrm{E}$ is administrated for NASH, treatment with lower dose (300-400 mg/day rather than $800 \mathrm{mg}$ ) of its agent should be considered [17].

Glutathione (GSH) Glutathione (GSH), L-glutamyl-Lcysteinyl-glycine, is a tripeptide present in every cell of the human body, and also has an anti-oxidative effect. A pilot study found that oral administration of GSH (300 mg/day) for 4 months can decrease ALT levels and hepatic steatosis in Japanese NAFLD patients, in those without severe fibrosis or uncontrolled diabetes. Large-scale clinical trials are needed to verify its efficacy [21].

UDCA Ursodeoxycholic acid (UDCA), which is covered by health insurance for chronic liver diseases in Japan, is known to have anti-oxidative efficacy [22]. According to a large, multicenter RCT, standard dose of UDCA has no effects on liver histology in NASH [23], although some studies suggest that high dosage of this agent may show a favorable effect. Currently, UDCA is not recommend for NASH treatment in the guidelines [5-8].

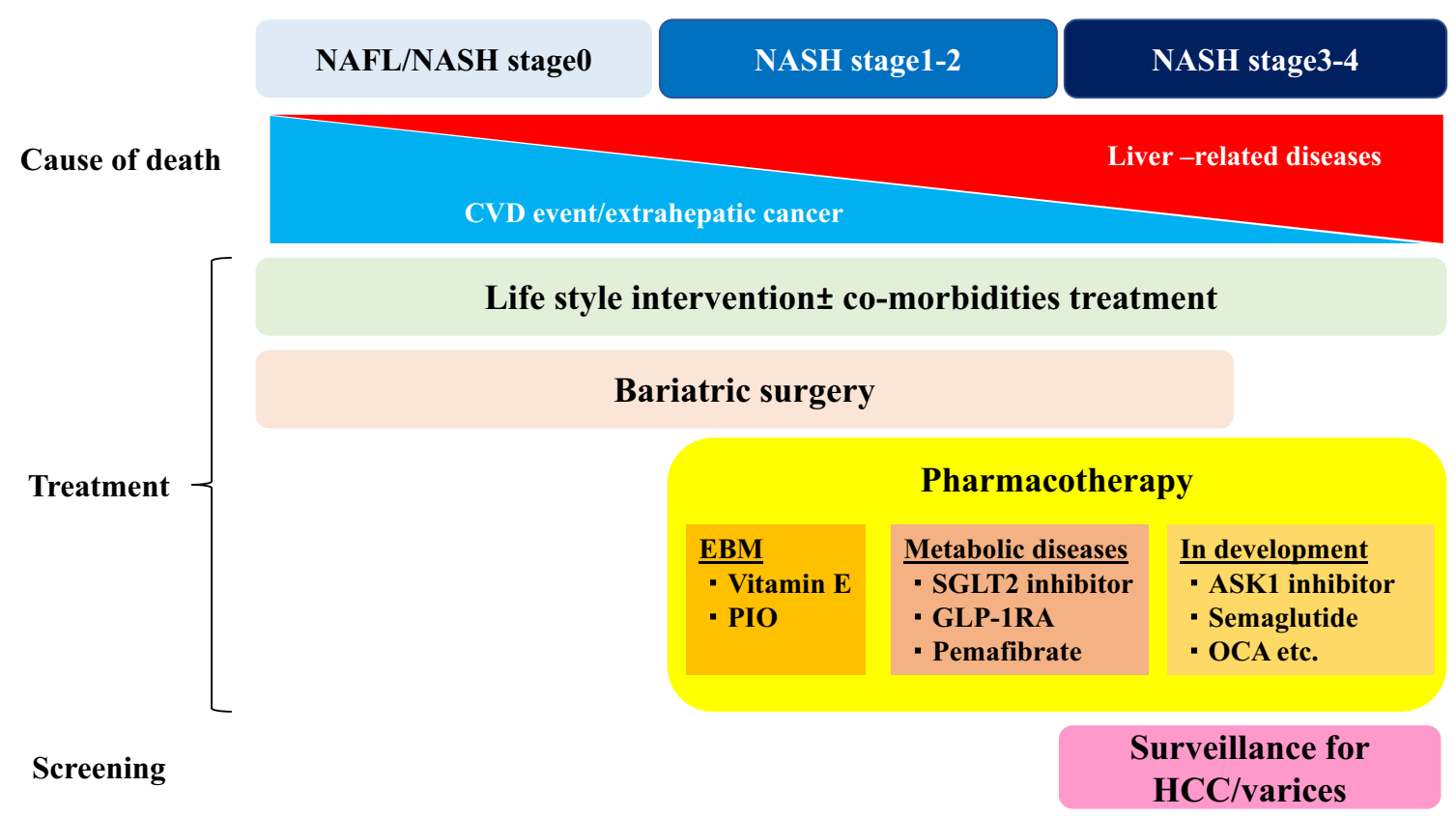

Fig. 1 Fibrosis stage-based treatment algorithm for NASH/NAFLD 


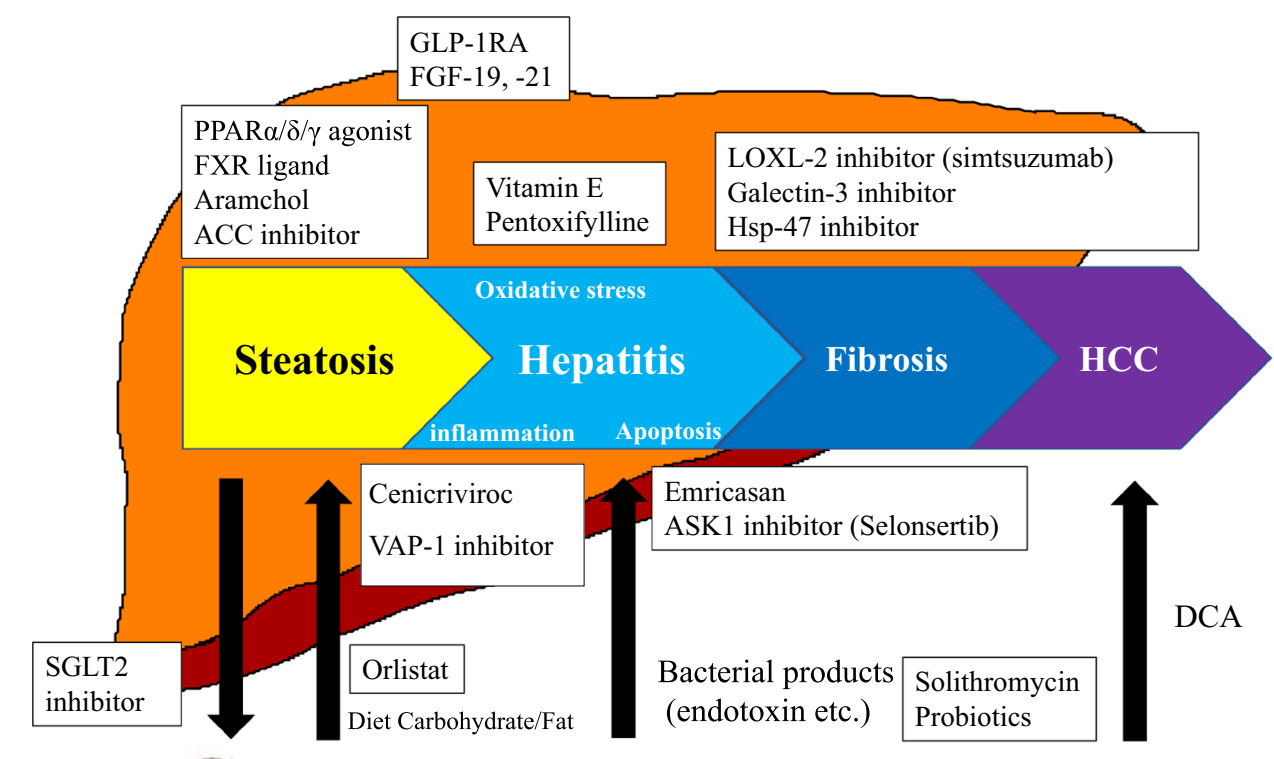

Glucose excretion
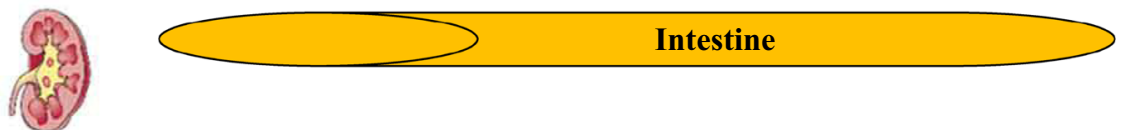

Fig. 2 Targets of upcoming therapies for NASH/NAFLD

Fig. 3 PPAR agonists for NASH/NAFLD

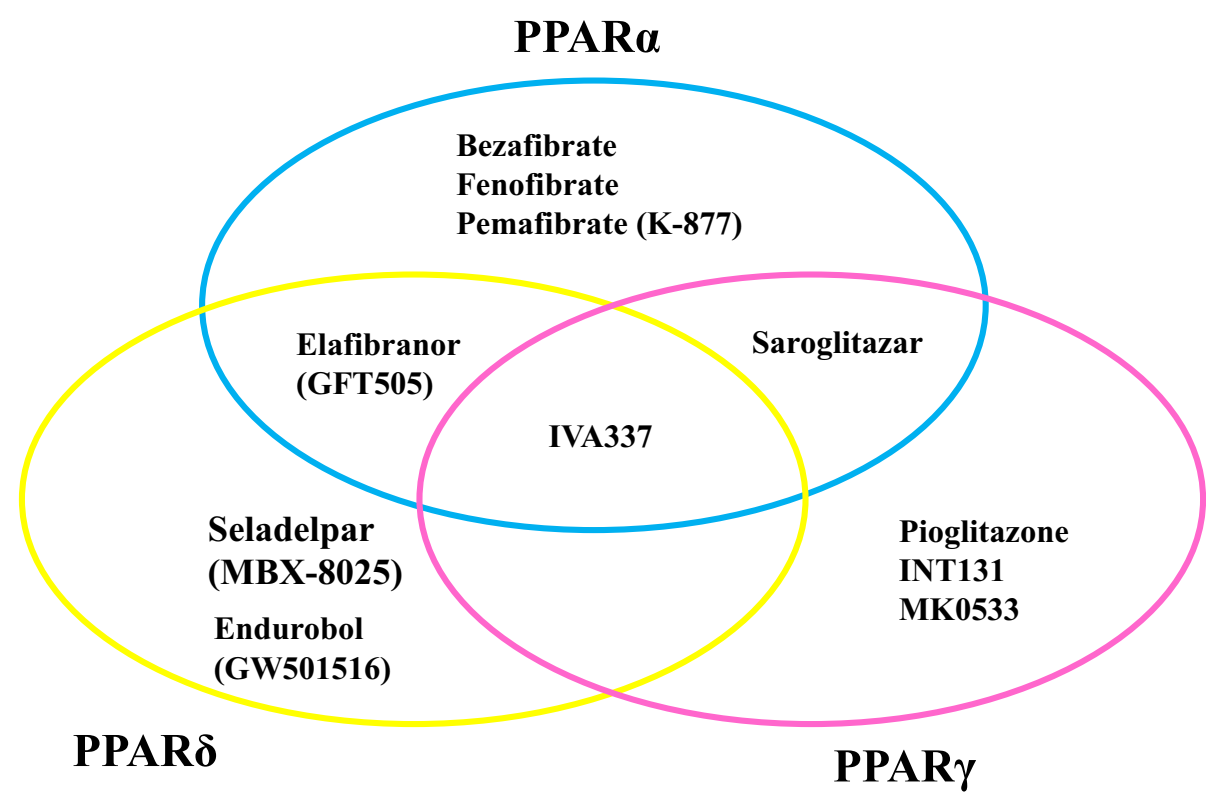

Peroxisome proliferator-activated receptor (PPAR) agonists (Fig. 3)

PPAR $\gamma$ Two randomized, double-blind, placebo-controlled trials (RDBPCT) have shown that pioglitazone [peroxisome proliferator-activated receptor gamma (PPAR $\gamma$ ) agonist] significantly ameliorated steatosis and necroinflammation compared to placebo in diabetic NASH [24, 25]. Recently, a 3-year study in $101 \mathrm{NASH}$ patients with prediabetes/T2DM (an 18-month RCT, followed by an 18-month open-label phase with pioglitazone treatment) confirmed its long-term safety and efficacy [26]. However, pioglitazone has also several concerns for wide clinical use, such as increased risks at prostate or pancreas cancer, body weight gain, fluid retention, bone fracture in women, and increased cardiovascular events. INT131, which is a selective PPAR $\gamma$ modulator (SPPARM $\gamma$ ), is in development for T2DM patients. INT131 demonstrated dose-dependent reductions in HbA1c, equivalent to $45 \mathrm{mg}$ pioglitazone, but with less fluid accumulation and weight 
gain [27]. Although no study with INT131 for the NASH treatment has been initiated, its agent will be expected in the future. MSDC-0602K is a PPAR $\gamma$-sparing thiazolidinedione. A study to evaluate the safety, tolerability, and efficacy of MSDC $0602 \mathrm{~K}$ in patients with NASH is ongoing (EMMINENCE). This is a RDBPCT of three doses of MSDC-0602K or placebo given orally once daily to subjects with biopsy-proven NASH with fibrosis and no cirrhosis (NCT02784444).

$P P A R \alpha$ There have been no studies proving the efficacy of PPAR $\alpha$ agonists, such as bezafibrate or fenofibrate, which are extensively used in the treatment of hypertriglyceridemia but have no impact in NASH/NAFLD. Bezafibrate has been believed to be effective in breast cancer patients with tamoxifen-induced NASH [28]. Pemafibrate (K-877), a selective PPAR $\alpha$ modulator (SPPARM $\alpha$ ), will be described as below.

Saroglitazar Saroglitazar is a dual PPAR $\alpha / \gamma$ agonist approved for the treatment of dyslipidemia in diabetic patients in India [29]. Phase 2 RDBPCT comparing three doses of saroglitazar (1, 2, or $4 \mathrm{mg}$ ) with placebo in NAFLD is now ongoing (EVIDENCES II; NCT03061721). The primary endpoint of the study is percentage change from baseline in serum ALT levels at week 16 in the saroglitazar groups as compared to the placebo group.

Elafibranor (GFT505) Elafibranor is an unlicensed dual agonist of PPAR $\alpha / \delta$ receptors, and has been shown to improve steatosis, inflammation, and fibrosis in mouse models of NAFLD [30]. A phase IIb RDBPCT showed patients resolving NASH without worsening hepatic fibrosis with $120 \mathrm{mg}$ elafibranor in those with $\mathrm{NAS} \geq 4$ (GOLDEN-505) [31]. Treatment was not effective in those with NAS $<4$ (19 vs. $12 \%, p=0.045)$. A multicenter, phase 3 RDBPCT study to evaluate the efficacy and safety of elafibranor in NASH without cirrhosis is ongoing (RESOLVE-IT) (NCT02704403). The primary objectives of this study are to evaluate the effect of elafibranor (120 mg/day) treatment in NASH patients (NAS $\geq 4$ ) with stage $2 / 3$ fibrosis compared to placebo on (1) histological improvement and (2) all-cause mortality and liver-related outcomes in patients with NASH and fibrosis. RESOLVEIT is a RDBPCT (2:1), conducted in approximately 2000 patients, at 250 centers worldwide.

Pan-PPAR agonist IVA337 is an anti-fibrotic treatment with a unique mechanism of action going through the activation of all three alpha, gamma, and delta PPARs (pan-PPAR agonist). IVA337 is effective in experimental skin fibrosis and lung fibrosis [32, 33]. A phase 2b RDBPCT in NASH to assess IVA337 is now recruiting (NATIVE). NATIVE study will investigate the safety and efficacy of two doses of IVA337 (800 and $1200 \mathrm{mg} /$ day) over a 24-week period and will enroll up to 225 patients in 12 European countries (NCT03008070).
Antidiabetic drugs

T2DM is strongly associated with NASH and liver-related mortality. The most important problem is what kinds of drugs are the most appropriate for NASH/NAFLD with diabetes among a variety of antidiabetic medications. Ideal anti-diabetic treatments on the view of NASH treatment must have weight-reducing efficacy, reduced cardiovascular event, prevention of HCC, low cost, and improved QOL [34]. There are no approved diabetic therapies except pioglitazone for NASH [5-8]. Metformin is now positioned as the first-line therapy according to the guideline of ADA/ EASD because of its low cost, weight-reducing effect, preventive effect on cardiovascular event, and safety profiles. Unfortunately, metformin has no data regarding improvement in liver enzymes and histology in NASH/ NAFLD, although it is associated with a reduced incidence of HCC and extrahepatic malignancies. Novel antidiabetic drugs will become a candidate for the treatment of NASH as we previously reviewed this point [34]. Incretin-associated drugs are classified into DPP4 inhibitors and glucagon-like peptide 1 receptor agonists (GLP-1RA). Unfortunately, there is conflicting evidence showing efficacy of DPP4 inhibitors in NASH/NAFLD patients with diabetes, although a number of patients involved into these studies is relatively small [34]. The efficacy of liraglutide, a GLP-1RA, was reported in NASH patients in Western countries (LEAN study [35]) and Japanese studies (LEANJ study [36]). That most of patients naïve to injection therapy will hesitate daily injection therapy. Dulaglutide has some advantages such as weekly injection, disposable and prefilled device, and similar safety profiles to other GLP-1RAs. Semaglutide, a novel GLP-1 RA, is in advanced stages of development for diabetes. To investigate the effect of semaglutide on NASH, a phase 2 RDBPCT comparing the efficacy and safety of three different doses of once-daily subcutaneous semaglutide versus placebo in 372 participants with NASH is now ongoing (NCT02970942). Semaglutide has three advantages over other GLP-1RA. First, the SUSTAIN-6 trial showed that semaglutide has a potential benefit on prevention of cardiovascular events [37]. Second, semaglutide is superior to dulaglutide on glucose control and weight loss in T2DM patients (SUSTAIN 7 trial). SUSTAIN 7 is a phase $3 b$, 40 -week, efficacy and safety trial of $0.5 \mathrm{mg}$ semaglutide versus $0.75 \mathrm{mg}$ dulaglutide and $1.0 \mathrm{mg}$ semaglutide versus $1.5 \mathrm{mg}$ dulaglutide, both once-weekly, as add-on to metformin in 1201 people with T2DM. Third, oral agent of semaglutide is now under development and of clinical use in the near future. As a result, among a variety of GLP-1 RA, dulaglutide or semaglutide will be the most promising in the treatment of diabetic NASH [34, 38]. According to the AASLD guidance, however, it is premature to consider 
GLP-1RA to specifically treat in NASH/NAFLD patients without diabetes [8] because of insufficient evidence. Subanalyses of three RDBPCT of SGLT2 inhibitor (canagliflozin [39, 40], luseogliflozin [41]) for the treatment of T2DM, serum transaminase activities in SGLT2 inhibitortreated patients were significantly reduced compared to those in the placebo group. The finding that causes of abnormal ALT level (31 IU/L or above) in a majority of Japanese diabetic patients may be associated with NAFLD [42] implies that the efficacy of SGLT2 inhibitor on NASH/NAFLD patients can be expected. Several pilot studies found significant reduction in ALT, body weight, and the fatty liver index in NAFLD patients [43-45]. The impact of SGLT2 inhibitor on liver histology is not confirmed. Takeda et al. reported a case of NASH with T2DM who resolved steatosis, inflammation, and hepatocyte ballooning after the ipragliflozin treatment [46]. Akuta et al. also recently demonstrated that all eight NAFLD patients with SGLT2 administration relieved hepatic steatosis and three of them obtained improvement in liver fibrosis [47]. Two open RCTs have been reported from Japan to compare the efficacy of SGLT2 inhibitor to other diabetic medications such as pioglitazone and metformin. The first repot is to compare the effect of luseogliflozin to metformin in T2DM patients with NAFLD. Hepatic steatosis, evaluated by liver-to-spleen $(L / S)$ ratio on $\mathrm{CT}$, was significantly reduced in the luseogliflozin group compared to in the metformin group [48]. The aim of another report is to compare the efficacy of ipragliflozin versus pioglitazone in NAFLD patients with T2DM. Serum ALT levels, $\mathrm{HbA}_{1 \mathrm{c}}$, and fasting plasma glucose were similarly reduced in the two treatment groups. Nevertheless, body weight and visceral fat area showed significant reductions only in the ipragliflozin group compared with the pioglitazone group [49]. A few open pilot studies of SGLT2 inhibitor in NAFLD patients are ongoing in the western countries (NCT02696941) or Asia (NCT02875821, NCT02964715). The effect of SGLT2 inhibitors versus other diabetic drugs (metformin, sulfonyl urea) is also investigated (NCT02696941, NCT02649465). The effects of empagliflozin treatment on hepatocellular lipid content, liver energy metabolism, and body composition is now investigated in a multicenter, RDBPCT, interventional, and exploratory pilot study in patients with newly diagnosed T2DM (NCT02637973).

\section{Lipid-altering agents}

Approved agents for dyslipidemia Ezetimibe, a potent inhibitor of cholesterol absorption, has been explored for the treatment of NASH/NAFLD, but conflicting results exist [50-52]. Histological findings (steatosis and inflammation) have been relived after the ezetimibe treatment without control arms [50, 51]. A RDBPCT (MOZART study) showed that ezetimibe $10 \mathrm{mg}$ orally daily for 24 weeks did not significantly affect hepatic steatosis over placebo [52]. A meta-analysis using six studies (two RCT and four pilot) has shown that ezetimibe may decrease serum liver enzymes and hepatic steatosis, but histological effectiveness remains uncertain [53]. Omega-3 fatty acids are often administrated for patients with hypertriglyceridemia. Two large studies (EPE-A study [54], WELCOME study [55]) failed to show the therapeutic benefit of omega-3 fatty acids in patients with NASH/NAFLD. Omega-3 fatty acids is limited to be used in NASH/ NAFLD with hypertriglyceridemia [8]. Since NAFLD patients are at high risk of cardiovascular morbidity or mortality, statins could be used to treat dyslipidemia with NASH/NAFLD [8]. Statin use seems to be associated with inhibition of hepatic inflammation, improvement of hepatic fibrosis, and reduced hepatocarcinogenesis [56], although prospective RCTs are now difficult to perform.

Pemafibrate Pemafibrate, a novel SPPARM $\alpha$, was approved in Japan in 2017. In Japan, phase 2, RDBPCT decreased serum transaminase activities as well as lipid profiles in patients with dyslipidemia without increasing adverse effects [57]. Pemafibrate, which improves liver pathology in diet-induced rodent model of NASH [58], will become a promising therapeutic agent for human NASH. In Japan, clinical trials for the treatment of NAFLD/NASH will begin in the near future.

Aramchol Aramchol, a cholic-arachidic acid conjugate, has inhibitory effects of stearoyl-CoA desaturase (SCD). Aramchol was initially produced for treatment of gallstone [59]. However, animal experiments showed a strong reduction of hepatic fat accumulation rather than gallstone dissolution. In humans, hepatic fat content was significantly reduced in the aramchol (300 mg/day) group [60]. Higher doses of aramchol (400 and $600 \mathrm{mg}$ ) are currently being tested on biopsy-proven NASH patients without cirrhosis in a 52-week phase $2 \mathrm{~b}$ trial, which evaluates their effect on hepatic triglyceride content using MR spectroscopy (NCT02279524).

GS0976 Acetyl-CoA carboxylase (ACC) is a key enzyme that regulates the conversion of malonyl-CoA to acetylCoA [61]. Malonyl-CoA is a key regulator of fatty acid metabolism, controlling the balance between de novo lipogenesis and fatty acid oxidation. An open-label, proofof-concept study evaluating GS-0976, an investigational inhibitor of ACC, in NASH patients. The data, from ten patients treated with GS-0976 $20 \mathrm{mg}$ taken orally once daily for 12 weeks, indicated that treatment was associated with statistically significant improvements in liver fat content and noninvasive markers of fibrosis (NCT02856555). At week 12, patients receiving GS-0976 experienced a 43\% median relative decrease in liver fat content, from 15.7 to 
$9.0 \%(p=0.006)$, as measured by magnetic resonance imaging-proton density fat fraction (MRI-PDFF). Median liver stiffness, a noninvasive marker of fibrosis, declined from 3.4 to $3.1 \mathrm{kPa}$ at week $12(p=0.049)$, as assessed by magnetic resonance elastography (MRE). In addition, patients with reductions in hepatic fat demonstrated improvements in liver biochemistry and serum markers of fibrosis and apoptosis, supporting the biological activity of GS-0976. A separate phase 2 RDBPCT evaluating GS-0976 in 126 patients with NASH is completed. According to Liver Meeting 2017, GS-0976 demonstreated signficant decrease in hepatic fat content and TIMP-1 (a serum marker associated with hepatic fibrosis).

\section{Anti-hypertensive drugs}

Angiotensinogen receptor blockers (ARB) There are no particularly favored agents for control of hypertension, although a few studies suggest that angiotensinogen receptor blockers (ARB) may have anti-fibrotic effects in NASH patients $[62,63]$. Unfortunately, a RDBPCT regarding the effect of losartan for 96 weeks in NASH patients failed because of slower recruitment than expected due to the widespread use of ARB in NASH patients [64]. It may be difficult to plan prospective RDBPCT to establish the efficacy or ARB for the treatment of NASH/NAFLD.

\section{FXR ligand}

Obeticholic acid (OCA) Obeticholic acid (OCA), a ligand of farnesoid X receptor (FXR), is a synthetic variant of natural bile acid chenodeoxycholic acid. In animal models, FXR activation has been demonstrated to reduce hepatic glucogenesis, lipogenesis, and steatosis. In the FLINT trial, treatment with OCA achieved a primary end-point of improving the necro-inflammation without worsening of fibrosis in $46 \%$ of the treated patients with NASH. Moreover, compared to placebo, NASH resolution was obtained in $22 \%$ of treated patients [65]. A phase 2 RDBPCT in Japan (FLINT-J trial) showed that high doses of OCA (40 mg/day) significantly resolved NASH compared with placebo (38 vs. $20 \%, p=0.049)$. Fibrosis improvement in the OCA treated group is similar to that in the placebo group. There are plausible reasons explaining this discrepancy between FLINT and FLINT-J study. In the FLINT-J study, NASH with mild fibrosis at entry is prevalent. Some patients in the OCA group refused post-treatment liver biopsy, and those are classified into non-responders. An international, phase 3 study (REGENERATE study) is now ongoing. However, OCA has several drawbacks, such as elevated LDL levels, itching, and high cost [65].

INT-767 INT-767 is a bile acid analogue that acts as a dual agonist on FXR/Takeda G-protein-coupled receptor 5
(TGR5). In an animal model, INT-767 improved histological features of NASH and modulated the activation of hepatic monocytes [66]. TGR5 has been known to affect energy metabolism, glucose homeostasis, bile composition/ secretion, and inflammation.

Non-bile acid FXR Selective non-bile acid synthetic FXR agonists have been developed to resolve disadvantage of OCA. Those have the potential to provide metabolic effects without increasing side effects of pruritus and elevated LDL. Phase 2 studies with GS-9674 are ongoing in patients with NASH (NCT02854605), primary biliary cholangitis (PBC), and primary sclerosing cholangitis (PSC). Two other FXR agonists, LMB763 (NCT02913105) and LJN452 (NCT02855164), have been developed and are in phase 2 trials.

$M G L-3196$ The thyroid hormone receptor $\beta$ (THR $\beta$ ) is the predominant liver thyroxine (T4) receptor, through which increased cholesterol metabolism and excretion through bile is mediated. MGL-3196, a highly selective THR $\beta$ agonist, has been developed to target dyslipidemia but has also been shown to reduce hepatic steatosis in fatfed rats [67]. Phase 2 trials are ongoing in patients with biopsy-proven NASH and $\geq 10 \%$ liver steatosis using percent change from baseline hepatic fat fraction assessed by MRI-PDFF as a primary outcome (NCT02912260).

\section{Anti-inflammatory and anti-apoptosis agents}

Pentoxifylline Pentoxifylline (PTX), a methylxanthine derivative, has anti-inflammatory effects and decreases oxidative stress. An RDBPCT showed that PTX therapy for 1 year significantly improved histological features of NASH compared to placebo [68]. A meta-analysis evaluating five studies showed improvement histological findings such as lobular inflammation and NAS without affecting lipid profiles [69]. However, this drug is no longer commercially available in Japan because its initial efficacy for treating after-effects of brain stroke was reevaluated and found to be insufficient.

Selonsertib Apoptosis signal-regulating kinase 1 (ASK1) is activated by extracellular TNF $\alpha$, intracellular oxidative or ER stress and initiates the p38/JNK pathway, resulting in apoptosis and fibrosis [70]. Inhibition of ASK1 has therefore been proposed as a target for the treatment of NASH. An open-label phase 2 trial evaluating the investigational ASK1 inhibitor selonsertib (GS-4997) alone or in combination with the monoclonal antibody simtuzumab (SIM) in NASH patients with moderate-to-severe liver fibrosis (stages 2/3). The data demonstrate regression in fibrosis that was, in parallel, associated with reductions in other measures of liver injury in patients treated with selonsertib for 24 weeks. Patients receiving selonsertib demonstrated improvements in several measures of liver 
disease severity, including fibrosis stage, progression to cirrhosis, liver stiffness (measured by MRE), and liver fat content (measured by MRI-PDFF). As no differences were observed between combination and monotherapy, results are presented for selonsertib (18 and $6 \mathrm{mg}$ ) with/without SIM and for SIM alone [71]. Thus, international phase 3 trials evaluating selonsertib among NASH patients with stage 3 (STELLAR3; NCT03053050) or cirrhosis (STELLAR4; NCT03053063) are ongoing (STELLAR program).

Tipelukast MN-001 (tipelukast) is a novel, orally bioavailable small-molecule compound that exerts its effects through several mechanisms to produce its antifibrotic and anti-inflammatory activity in preclinical models, including leukotriene (LT) receptor antagonism, inhibition of phosphodiesterases (PDE) (mainly 3 and 4), and inhibition of 5-lipoxygenase (5-LO). An open-label study to evaluate the efficacy, safety, tolerability, and PK of MN001 (Tipelukast) on HDL function and serum triglyceride levels in NASH/NAFLD with hypertriglyceridemia is ongoing (NCT02681055).

Emricasan Emricasan, an irreversible caspase inhibitor, improves NAS and fibrosis in murine models of NASH [72]. A phase $2 \mathrm{~b}$ study in patients with NASH (stage 1-3) is evaluating the efficacy of 72 weeks of emiricasan 10 or $100 \mathrm{mg}$ (ENCORE-NF, NCT02686762). Another phase 2b study in patients with NASH with cirrhosis and severe portal hypertension is assessing the efficacy of three doses Emricasan (10, 50, $100 \mathrm{mg} /$ day) on portal hypertension (ENCORE-PH, NCT02960204). Primary outcome is mean change in hepatic venous pressure gradient (HVPG).

Vascular adhesion protein-1 (VAP-1) inhibitor (BI 1467335) The adhesion molecule vascular adhesion protein-1 (VAP-1) is a membrane-bound amine oxidase that promotes leukocyte recruitment to the liver, and the soluble form (sVAP-1) accounts for most circulating monoamine oxidase activity, has insulin-like effects, and can initiate oxidative stress [73]. VAP-1 is directly involved in stellate cell activation and is a strong profibrogenic stimulus. Thus, targeting VAP-1 may result in a decrease in leukocyte recruitment and reduction of inflammation and fibrosis. BI 1467335 is a VAP-1 inhibitor that works by blocking leucocyte adhesion and tissue infiltration in inflammatory process. Phase 2a trial of BI 1467335 is a multicenter, RDBPCT in 150 patients with clinical evidence of NASH (NCT03166735).

\section{Gut microbiome}

IMM-124e IMM-24e is an IgG-rich extract to bovine colostrum from cows immunized against lipopolysaccharide (LPS). IMM-24e can reduce exposure of the liver to gut-derived bacterial products and LPS. An open-label, phase $1 / 2$ clinical trial in ten patients with biopsy-proven
NASH improved liver enzymes as well as glycemic control via increase in serum levels of GLP-1, adiponectin, and T regulatory cells [74]. A phase 2 RDBPCT of IMM-124E for 24 weeks is currently ongoing for $\mathrm{NASH}$ patients (NCT02316717).

Solithromycin Solithromycin is a highly potent nextgeneration macrolide antibiotic. In a phase 2 open-label study, all six NASH patients had reductions in NAS (mean reduction, 1.3) and ALT level (mean reduction, 17.8 U/L) after 90 days of treatment with solithromycin (NCT02510599).

TLR4 antagonist JKB-121 is a long-acting small molecule that is efficacious as a weak antagonist at the Toll-like receptor 4 (TLR4). It is a non-selective opioid antagonist that has been shown to prevent the LPS-induced inflammatory liver injury in a methionine/choline-deficient diet fed rat model of NAFLD. In vitro, JKB-121 neutralized or reduced the LPS-induced release of inflammatory cytokines, deactivated hepatic stellate cells, inhibited hepatic stellate cell proliferation, and collagen expression. Inhibition of the TLR4 signaling pathway may provide an effective therapy in the prevention of inflammatory hepatic injury and hepatic fibrosis in NASH patients [75]. A phase 2 RDBPCT trial of JKB-121 for the treatment of NASH is ongoing (NCT02442687).

\section{Antifibrotic agents}

Given that hepatic fibrosis stage is the most important determinant of mortality in NASH patients [9, 76], there is an unmet medical need for an effective anti-fibrotic treatment for those with advanced fibrosis. Several anti-fibrotic agents have been developed for the treatment of advanced NASH.

Cenicriviroc Cenicriviroc (CVC), a C-C motif chemokine receptor-2/5 (CCR2/5) antagonist, has been developed to primarily target inflammation. This agent has also antifibrotic effects and improves insulin sensitivity. Macrophage recruitment through CCR2 into adipose tissue is believed to play a role in the development of insulin resistance and T2DM. Administration of CCR2 antagonist resulted in modest improvement in glycemic parameters compared with placebo [77]. CCR5 antagonist is expected to impair the migration, activation, and proliferation of collagen-producing hepatic stellate cells [78]. According to phase $2 b$ trial (CENTAUR study), significant improvement of fibrosis without worsening NASH after 1 year of CVC treatment was found (20\%) compared with placebo $(10 \%)$ [79]. Although asymptomatic amylase elevation (grade 3) was more frequent in the CVC group than in the placebo group, this agent is well tolerated. Phase 3 evaluation for the treatment of NASH with stage $2 / 3$ fibrosis is now ongoing and recruiting (AURORA study; NCT03028740). 
Simtuzumab (SIM) SIM is a monoclonal antibody against the enzyme lysyl oxidase-like 2 (LOXL-2) responsible for the cross-linking of collagen and overexpressed during the fibrosis progression [80]. Unfortunately, this agent could not bring additional benefit over ASK1 inhibitor to improve hepatic fibrosis in phase $2 \mathrm{~b}$ study as mentioned above. Finally, SIM was withdrawn from candidates of NASH treatments.

Galectin-3 antagonist Galectin-3 protein expression, which is essential to the development of hepatic fibrosis, was increased in NASH with the highest expression in macrophages surrounding lipid-laden hepatocytes. In mice models, GR-MD-02, a galectin-3 inhibitor, resulted in marked improvement in liver histology with a significant reduction in NASH activity and collagen deposition [81]. Although there was no safety concern in phase $2 \mathrm{a}$ trials in NASH patients with stage 3 fibrosis [82], there was no apparent improvement in the three non-invasive tests for assessment of liver fibrosis. A phase $2 \mathrm{~b}$ clinical trial to evaluate the safety and efficacy of GR-MD-02 for the treatment of liver fibrosis and resultant portal hypertension in 162 patients with NASH cirrhosis (NASH-CX trial) is now ongoing (NCT02462967). Top-line results will be reported in early December 2017.

ND-LO2-s0201 Hsp47 (heat shock protein 47) is a collagen-specific molecular chaperone that is essential for the maturation and secretion of collagen. ND-LO2-s0201 is a vitamin A-coupled lipid nanoparticle containing siRNA against HSP47. A phase 1 open study is completed to evaluate in subjects with severe hepatic fibrosis (stage 3/4) (NCT02227459).

\section{Ongoing or scheduled clinical trials in Japan}

To our best knowledge, there are several ongoing or scheduled clinical trials in Japan. Two agents, including semaglutide and ASK1 inhibitor, were already mentioned above.

Nonsteroidal MRA Several nonsteroidal antagonists of the mineralocorticoid receptor (MRA) are in clinical development with a clear focus on the treatment of diabetic kidney diseases. In Japan, MT3995, a novel nonsteroidal MRA, is currently tested for the treatment of NASH (phase 2, NCT02923154).

FGF-21 (fibroblast growth factor-21) Fibroblast growth factor 21 (FGF-21), a hepatokine, is a 181-aminoacid-secreted protein that is produced in the liver. FGF-21 regulates glucose in the liver and the white adipose tissue and its circulating levels are elevated in NAFLD patients, considered to play a protective role against NAFLD [83]. A RCT in a small group of obese T2DM patients with FGF-21 found significant improvement in lipid profiles as well as weight loss, reduced insulin levels, and raised adiponectin [84]. A phase 2 study of BMS-986036, a recombinant FGF-21 in NASH patients for 16 weeks, is completed (NCT02413372). This was a multicenter RDBPCT (1:1:1) in adults with BMI $\geq 25 \mathrm{~kg} / \mathrm{m}^{2}$, biopsyproven NASH with stage 1-3, and hepatic fat fraction $\geq 10 \%$, assessed by MRI-PDFF. Patients received subcutaneous injections of BMS-986036 $10 \mathrm{mg}$ daily $(n=25)$, BMS-986036 $20 \mathrm{mg}$ weekly $(n=23)$, or placebo $(n=26)$ daily for 16 weeks. The primary efficacy endpoint was absolute change in MRI-PDFF at week 16. At week 16, both dosing regimens of BMS-986036 (10 mg daily or $20 \mathrm{mg}$ weekly) significantly reduced liver fat as measured by MRI-PDFF versus placebo (6.8 and $5.2 \%$, respectively, Z. $1.3 \%, p=0.0004$ and $p=0.008)$. Both dosing regimens also improved Pro-C3 (N-terminal type III collagen propeptide, a fibrosis biomarker [85]), liver stiffness evaluated by MRE, as well as adiponectin, ALT, and AST. Improvements in lipid profiles were also observed in the treatment groups. Overall, BMS-986036 had a favorable safety profile, with no deaths or serious adverse events related to treatment, and no discontinuations due to adverse events. An international phase 3 study of BMS-986036 for the treatment of NASH with stage $3 / 4$ will be planned.

\section{Drug repositioning}

Amlexanox Amlexanox is an inhibitor of noncanonical IкB kinases IKK- $\varepsilon$ and TANK-binding kinase 1. Amlexanox is an approved small-molecule therapeutic presently used in the clinic to treat aphthous ulcers and asthma. Treatment of obese mice with amlexanox elevates energy expenditure through increased thermogenesis, producing weight loss, improved insulin sensitivity, and decreased steatosis. Because of its record of safety in patients, amlexanox may be an interesting candidate for clinical evaluation in the treatment of NAFLD [86]. An open-label study and a phase 2 RDBPCT are currently assessing the effects of 12 weeks of amlexanox in patients with diabetes, obesity, and fatty liver on hepatic fat content by MRI, HbA1c, and weight (NCT01975935 and NCT01842282).

Pirfenidone Pirfenidone (PFD) is an orally bioavailable pyridone derivative that has been clinically used for the treatment of idiopathic pulmonary fibrosis [87]. PFD markedly attenuated liver fibrosis in Western diet (WD)fed melanocortin 4 receptor-deficient (MC4R-KO) mice without affecting metabolic profiles or steatosis. PFD prevented liver injury and fibrosis associated with decreased apoptosis of liver cells in WD-fed MC4R-KO mice [88]. PFD can be repositioned as an antifibrotic drug for human $\mathrm{NASH}$. 


\section{Milestones in the treatment of NASH/NAFLD}

Until now, the gold standard of assessment for treatment efficacy in NASH has been liver histology. However, repeated liver biopsies are practically difficult to be performed in NASH patients, because of risk, sampling error, observers' variability of pathological interpretation, and cost. Simple, reliable, and cost-effective parameters should be established to monitor the disease and evaluate the treatment efficacy.

$A L T$ In the sub-analysis of PIVENS study, ALT response, which was defined as ALT reduction over $30 \%$ from baseline or ALT levels less than 40 IU/l, reflect histological improvement [89]. As we also previously reported in 2015 [90], ALT response was the best predictor of reduction in NAS or fibrosis regression in 52 Japanese patients with NASH undergoing repeated biopsies.

Body weight Weight loss has been believed to be associated with improvements of liver histology in patients with NAFLD/NASH. Data from 261 NASH patients receiving repeated liver biopsies showed that weight loss, the absence of diabetes, ALT normalization, and baseline NAS less than 5 were independent predictors of NASH resolution without fibrosis worsening after 1 year of lifestyle intervention [91].

HbAlc Decreased levels in HbA1c [92] were more strongly associated with fibrosis improvement in 39 Japanese patients with diabetes and NAFLD who underwent sequential liver biopsies. As a result, we believe that these three clinical parameters, including ALT, body weight, and HbA1c (ABC), can become the milestones in the treatment of NASH (Fig. 4), although the appropriate goal of each parameter to ameliorate hepatic fibrosis will be established.

FIB4 index Fibrosis 4 (FIB-4) index and NALD fibrosis score (NFS) are now established as the best predictors of severe fibrosis in NAFLD [8, 93-95]. FIB-4 index is simply calculated using an algorithm based on AST, ALT, platelet count, and age. The FLINT study demonstrated that OCA treatment of NASH patients led to a statistically significant decrease in FIB4 index from baseline as compared to placebo (OCA: -0.246 vs. placebo: -0.047 ; $p=0.0076$ ). Further, a decline in FIB-4 of $10 \%$ after 24 weeks of treatment predicted improvement in fibrosis by at least one stage as assessed by biopsy at 72 weeks $(p=0.0448)$. While NFS declined in the OCA-treated patients and increased in the placebo patients, it did not appear to be sensitive to changes in fibrosis. This result, presented by Dr. Sanyal in the annual meeting of AASLD held in 2015, provides support for the use of FIB-4 index as potential non-invasive alternative means for monitoring fibrosis changes in response to treatment (Fig. 4).

Imaging Although imaging studies such as VCTE (Fibroscan) [96-98] and MR elastography (MRE) [99] have

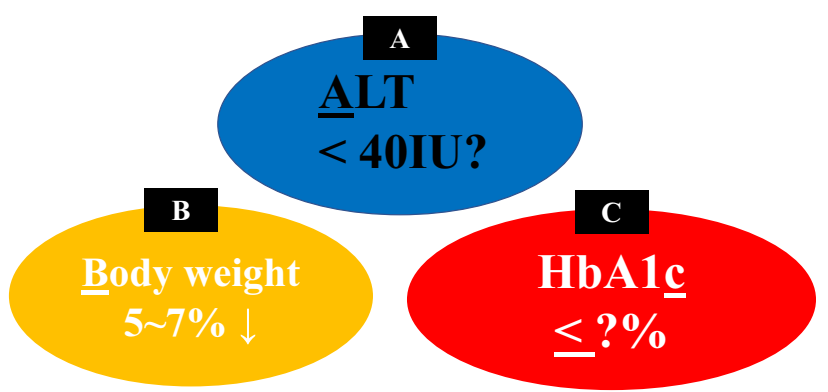

Fig. 4 Milestones in the treatment of NASH are "ABC"??

been extensively studied to detect severe fibrosis in NAFLD $[8,95]$, it is unknown whether these modalities are also useful to evaluate treatment efficacy. Many ongoing clinical trials, which evaluate clinical efficacy of NASH/ NAFLD, are using imaging modalities using MRI-PDFF/ MRE, which will become an alternative to liver biopsy.

\section{Who should treat?}

Diabetes specialist should pay attention to liver status. Three studies using Fibroscan showed that $12-18 \%$ of diabetic patients are estimated to have significant liver fibrosis by different cutoffs [100-102]. Another study using MRE found that advanced fibrosis (defined as MRE $\geq 3.6 \mathrm{kPa}$ ) was $7.1 \%$ in diabetic patients [103]. In a cross-sectional multicenter study conducted by JSGNAFLD, the presence of diabetes is associated with advanced fibrosis in 1365 biopsy-proven NAFLD patients [104]. These results imply the importance of collaboration between the hepatologists and diabetes team. However, the leading cause of mortality in patients with NAFLD is cardiovascular diseases, followed by extrahepatic cancer and liver-related diseases [105]. There are a variety of stakeholders in the treatment of NASH/NAFLD, including the hepatologist, cardiologist, endocrinologist, dietician, and patients (Fig. 5). Lifestyle modification intervention and pharmacotherapies should be delivered in collaboration with multi-disciplinary medical staff [106] (Fig. 5).

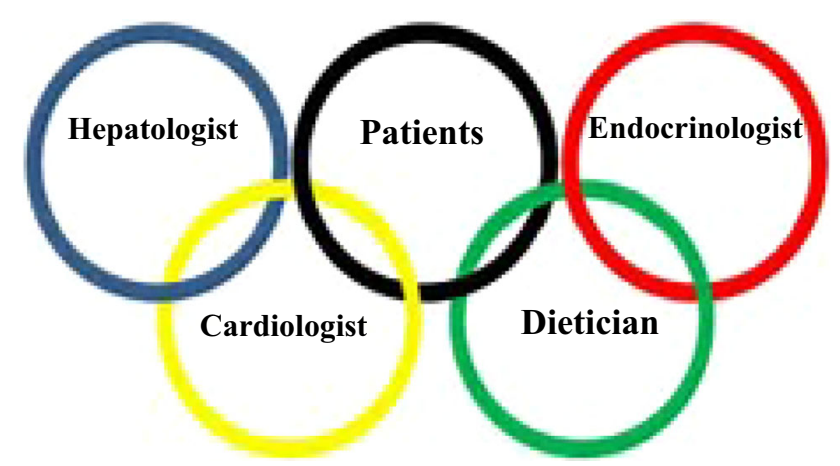

Fig. 5 Variety of stakeholders in the treatment of NASH/NAFLD 


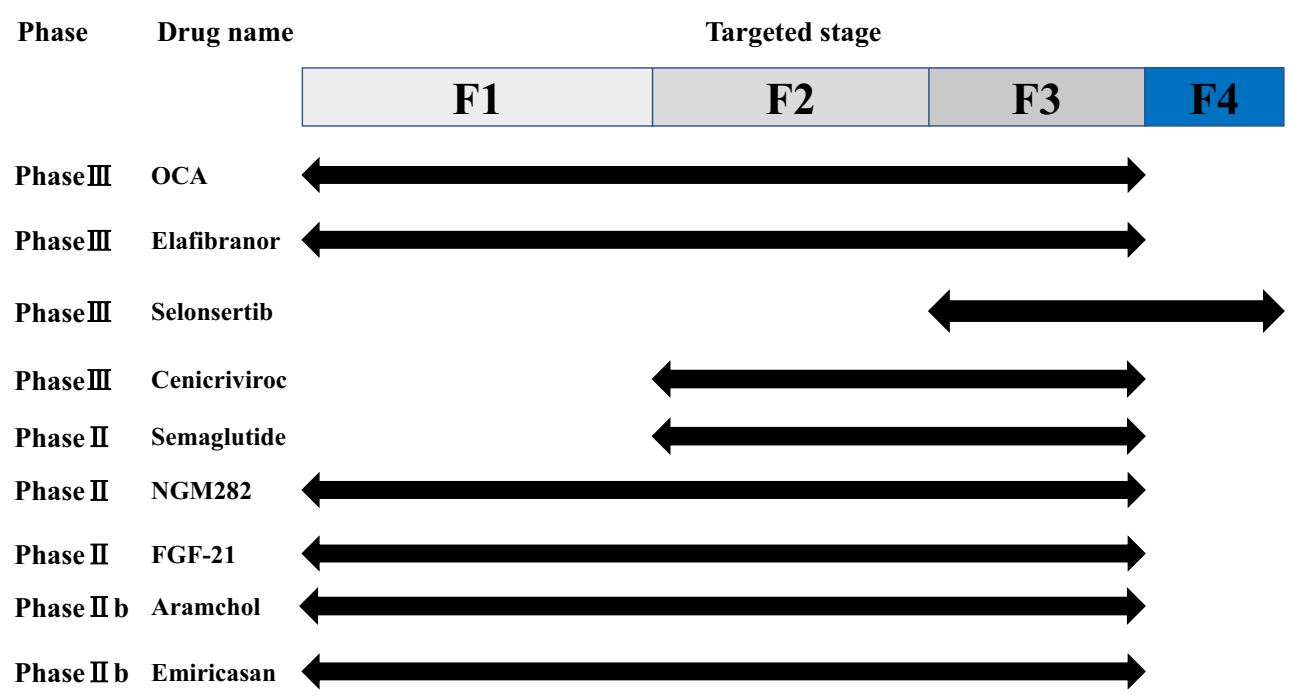

Fig. 6 NASH drug pipelines

Table 2 Clinically meaningful outcomes in patients with NAFLD/ NASH

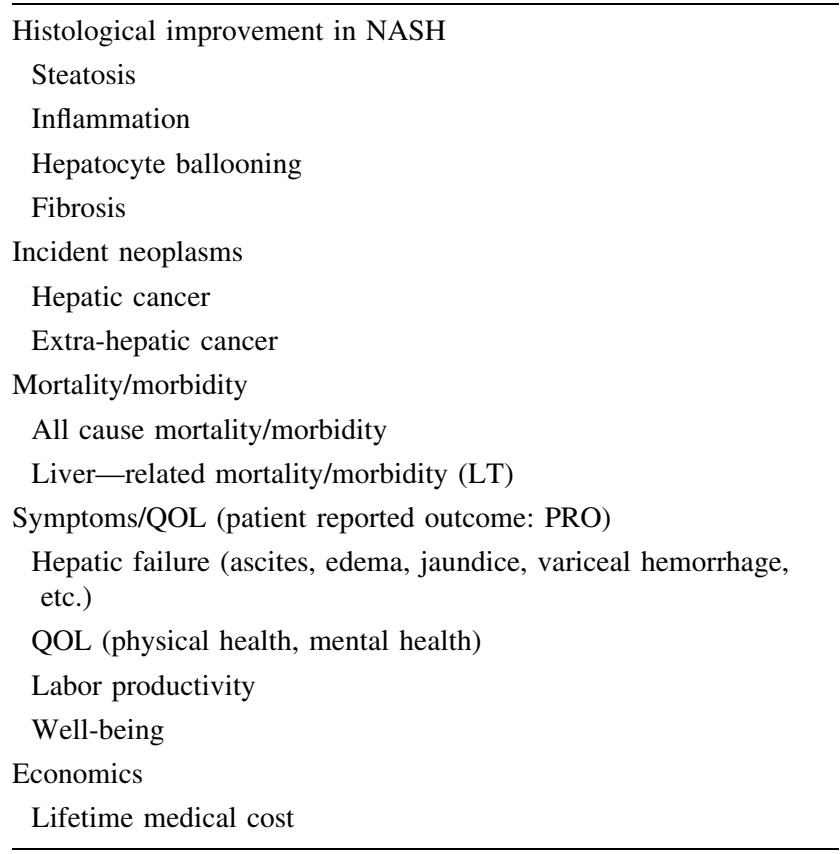

\section{Future perspectives}

Clinically meaningful outcomes in patients with NAFLD/ NASH are shown in Table 2. The primary endpoint should be measurable, sensitive to change, clinically meaningful, and be able to be quantified consistently. Reversal of steatohepatitis with at least no worsening of fibrosis has been considered as a primary endpoint of clinical trials.

Although hepatic fibrosis is the most critical determinant of all-cause or liver-related mortality in NASH, it remains to be solved whether improvement in hepatic fibrosis can lead to prevention of liver-related mortality in NASH patients. The prevalence of obesity and diabetes is dramatically increasing worldwide. NASH-related liver diseases (HCC, hepatic failure, and variceal hemorrhage) will soon be the leading causes of liver transplantation. It is estimated that the market size of NASH will reach 49 billion in 2027 in US, Japan, and EU 5 (England, France, Germany, Italy, and Spain). When pharmacotherapies are initiated for the target of liver disease in NASH/NAFLD, benefits and risks should be discussed with patients [8]. Patient-reported outcome (PRO) should be evaluated before and after pharmacotherapies. It is estimated that the risk for HCC development in NASH/NAFLD without advanced fibrosis is very small given the extremely large number of patients without advanced fibrosis within the general population. Economical cost and benefit should also be balanced [107].

\section{Conclusions}

To prevent liver-related morbidity/mortality in NASH patients, those with fibrosis should be considered for pharmacotherapies in addition to conventional dietary interventions. The first-line therapy for those without diabetes is vitamin $\mathrm{E}$ on the basis of accumulating evidence, although its impact on preventive effect of hepatic fibrosis and hepatocarcinogenesis remain uncertain. Diabetic NASH patients should be preferentially treated with novel drugs licensed for diabetes treatment such as GLP-1RA and SGLT2 inhibitors. SPPARM $\alpha$ (pemafibrate) is promising in NASH patients with dyslipidemia. There are currently several innovative agents in the drug pipeline for NASH worldwide. Four drugs (OCA, elafibranor, selonsertib, and 
CVC) have entered phase 3 trials (Fig. 6). Cost-effectiveness data and patient-centered benefits are also required to position their medications in the practical guidelines of NASH/NAFLD.

\section{Compliance with ethical standards}

Conflict of interest Authors must indicate whether or not they have/ had a financial relationship (within the last 3 years) with any organization that sponsored the research. They should also confirm that they have full control of all primary data and that they agree to allow the journal to review their data if requested.

Open Access This article is distributed under the terms of the Creative Commons Attribution 4.0 International License (http://crea tivecommons.org/licenses/by/4.0/), which permits unrestricted use, distribution, and reproduction in any medium, provided you give appropriate credit to the original author(s) and the source, provide a link to the Creative Commons license, and indicate if changes were made.

\section{References}

1. Younossi ZM, Koenig AB, Abdelatif D, et al. Global epidemiology of nonalcoholic fatty liver disease-meta-analytic assessment of prevalence, incidence, and outcomes. Hepatology. 2016;64:73-84.

2. Eguchi Y, Hyogo H, Ono M, et al; JSG-NAFLD. Prevalence and associated metabolic factors of nonalcoholic fatty liver disease in the general population from 2009 to 2010 in Japan: a multicenter large retrospective study. J Gastroenterol. 2009;2012(47):586-95.

3. Goldberg D, Ditah IC, Saeian K, et al. Changes in the prevalence of hepatitis $\mathrm{C}$ virus infection, nonalcoholic steatohepatitis, and alcoholic liver disease among patients with cirrhosis or liver failure on the waitlist for liver transplantation. Gastroenterology. 2017;152:1090-9.

4. Nakamura J, Kamiya H, Haneda M, et al. Causes of death in Japanese patients with diabetes based on the results of a survey of 45,708 cases during 2001-2010: report of the committee on causes of death in diabetes mellitus. J Diabetes Invest. 2017;8:397-410.

5. Chalasani N, Younossi Z, Lavine JE, et al; American Gastroenterological Association, American Association for the Study of Liver Diseases, American College of Gastroenterology. The diagnosis and management of non-alcoholic fatty liver disease: practice guideline by the American Gastroenterological Association, American Association for the Study of Liver Diseases, and American College of Gastroenterology. Gastroenterology. 2012;142:1592-609.

6. Watanabe S, Hashimoto E, Ikejima K, Japanese Society of Gastroenterology, Japan Society of Hepatology. Evidence-based clinical practice guidelines for nonalcoholic fatty liver disease/ nonalcoholic steatohepatitis. J Gastroenterol. 2015;50:364-77.

7. European Association for the Study of the Liver (EASL), European Association for the Study of Diabetes (EASD), European Association for the Study of Obesity (EASO). EASLEASD-EASO clinical practice guidelines for the management of non-alcoholic fatty liver disease. J Hepatol. 2016;64:1388-402.

8. Chalasani N, Younossi Z, Lavine JE, et al. The diagnosis and management of nonalcoholic fatty liver disease: practice guidance from the American Association for the Study of Liver Diseases. Hepatology (In press).
9. Dulai PS, Singh S, Patel J, et al. Increased risk of mortality by fibrosis stage in nonalcoholic fatty liver disease: systematic review and meta-analysis. Hepatology. 2017;65:1557-65.

10. Sumida Y, Niki E, Naito Y, Yoshikawa T. Involvement of free radicals and oxidative stress in NAFLD/NASH. Free Radic Res. 2013;47:869-80.

11. Sumida Y, Nakashima T, Yoh T, et al. Serum thioredoxin levels as a predictor of steatohepatitis in patients with nonalcoholic fatty liver disease. J Hepatol. 2003;38:32-8.

12. Hasegawa T, Yoneda M, Nakamura K, Makino I, Terano A. Plasma transforming growth factor-beta1 level and efficacy of alpha-tocopherol in patients with non-alcoholic steatohepatitis: a pilot study. Aliment Pharmacol Ther. 2001;15:1667-72.

13. Yoneda M, Hasegawa T, Sato K. Vitamin E therapy for NAFLD/NASH. Nutrition. 2015;31:898-9.

14. Sanyal AJ, Chalasani N, Kowdley KV, et al; NASH CRN. Pioglitazone, vitamin $\mathrm{E}$, or placebo for nonalcoholic steatohepatitis. N Engl J Med. 2010;362:1675-85.

15. Sato K, Gosho M, Yamamoto T, et al. Vitamin E has a beneficial effect on nonalcoholic fatty liver disease: a meta-analysis of randomized controlled trials. Nutrition. 2015;31:923-30.

16. Sumida $Y$, Naito $Y$, Tanaka $S$, et al. Long-term ( $\geq 2$ year) efficacy of vitamin $\mathrm{E}$ for non-alcoholic steatohepatitis. Hepatogastroenterology. 2013;60:1445-50.

17. Rinella ME, Sanyal AJ. Management of NAFLD: a stage-based approach. Nat Rev Gastroenterol Hepatol. 2016;13:196-205.

18. Miller ER 3rd, Pastor-Barriuso R, Dalal D, et al. Meta-analysis: high-dosage vitamin E supplementation may increase all-cause mortality. Ann Intern Med. 2005;142:37-46.

19. Lippman SM, Klein EA, Goodman PJ, et al. Effect of selenium and vitamin $\mathrm{E}$ on risk of prostate cancer and other cancers: the selenium and vitamin $\mathrm{E}$ cancer prevention trial (SELECT). JAMA. 2009;301:39-51.

20. Schürks M, Glynn RJ, Rist PM, et al. Effects of vitamin E on stroke subtypes: meta-analysis of randomised controlled trials. BMJ. 2010;4(341):c5702.

21. Honda Y, Kessoku T, Sumida Y, et al. Efficacy of glutathione for the treatment of nonalcoholic fatty liver disease: an openlabel, single-arm, multicenter, pilot study. BMC Gastroenterol. 2017; 17:96

22. Mitsuyoshi H, Nakashima T, Sumida Y, et al. Ursodeoxycholic acid protects hepatocytes against oxidative injury via induction of antioxidants. Biochem Biophys Res Commun. 1999;263:537-42.

23. Lindor KD, Kowdley KV, Heathcote EJ, et al. Ursodeoxycholic acid for treatment of nonalcoholic steatohepatitis: results of a randomized trial. Hepatology. 2004;39:770-8.

24. Belfort R, Harrison SA, Brown K, et al. A placebo controlled trial of pioglitazone in subjects with nonalcoholic steatohepatitis. N Engl J Med. 2006;355:2297-307.

25. Aithal GP, Thomas JA, Kaye PV, et al. Randomized, placebocontrolled trial of pioglitazone in nondiabetic subjects with nonalcoholic steatohepatitis. Gastroenterology. 2008;135:1176-84.

26. Cusi K, Orsak B, Bril F, et al. Long-term pioglitazone treatment for patients with nonalcoholic steatohepatitis and prediabetes or type 2 diabetes mellitus: a randomized trial. Ann Intern Med. 2016;165:305-15.

27. DePaoli AM, Higgins LS, Henry RR, et al; INT131-007 Study Group. Can a selective PPAR $\gamma$ modulator improve glycemic control in patients with type 2 diabetes with fewer side effects compared with pioglitazone? Diabetes Care. 2014;37:1918-23.

28. Saibara T, Onishi S, Ogawa Y, et al. Bezafibrate for tamoxifeninduced non-alcoholic steatohepatitis. Lancet. 1999;353:1802.

29. Shetty SR, Kumar S, Mathur RP, et al. Observational study to evaluate the safety and efficacy of saroglitazar in Indian diabetic dyslipidemia patients. Indian Heart J. 2015;67:23-6. 
30. Staels B, Rubenstrunk A, Noel B, et al. Hepatoprotective effects of the dual peroxisome proliferator-activated receptor alpha/ delta agonist, GFT505, in rodent models of nonalcoholic fatty liver disease/nonalcoholic steatohepatitis. Hepatology. 2013;58:1941-52.

31. Ratziu V, Harrison SA, Francque S, et al; GOLDEN-505 Investigator Study Group. Elafibranor, an agonist of the peroxisome proliferator-activated receptor- $\alpha$ and $-\delta$, induces resolution of nonalcoholic steatohepatitis without fibrosis worsening. Gastroenterology. 2016;150:1147-59.

32. Ruzehaji N, Frantz C, Ponsoye M, et al. Pan PPAR agonist IVA337 is effective in prevention and treatment of experimental skin fibrosis. Ann Rheum Dis. 2016;75:2175-83.

33. Avouac J, Konstantinova I, Guignabert C, et al. Pan-PPAR agonist IVA337 is effective in experimental lung fibrosis and pulmonary hypertension. Ann Rheum Dis. 2017;76:1931-40

34. Sumida Y, Seko Y, Yoneda M; Japan Study Group of NAFLD (JSG-NAFLD). Novel antidiabetic medications for nonalcoholic fatty liver disease with type 2 diabetes. Hepatol Res. 2017;47:266-80

35. Armstrong MJ, Gaunt P, Aithal GP, et al. Liraglutide safety and efficacy in patients with non-alcoholic steatohepatitis (LEAN): a multicentre, double-blind, randomised, placebo-controlled phase 2 study. Lancet. 2016;387:679-90.

36. Eguchi Y, Kitajima Y, Hyogo H, et al. Pilot study of liraglutide effects in non-alcoholic steatohepatitis and non-alcoholic fatty liver disease with glucose intolerance in Japanese patients (LEAN-J). Hepatol Res. 2015;45:269-78.

37. Marso SP, Bain SC, Consoli A, et al; SUSTAIN-6 Investigators. Semaglutide and cardiovascular outcomes in patients with type 2 diabetes. N Engl J Med. 2016;375:1834-44.

38. Katsiki N, Athyros VG, Karagiannis A, et al. Semaglutide, lipidlowering drugs, and NAFLD. Lancet Diabetes Endocrinol. 2017;5:329-30.

39. Seko Y, Sumida Y, Sasaki K, et al. Effects of canagliflozin, an SGLT2 inhibitor, on hepatic function in Japanese patients with type 2 diabetes mellitus: pooled and subgroup analyses of clinical trials. J Gastroenterol (In press).

40. Leiter LA, Forst T, Polidori D, et al. Effect of canagliflozin on liver function tests in patients with type 2 diabetes. Diabetes Metab. 2016;42(1):25-32.

41. Seino Y, Sasaki T, Fukatsu A, et al. Efficacy and safety of luseogliflozin as monotherapy in Japanese patients with type 2 diabetes mellitus: a randomized, double-blind, placebo-controlled, phase 3 study. Curr Med Res Opin. 2014;30:1245-55.

42. Shima T, Uto H, Ueki K, et al. Clinicopathological features of liver injury in patients with type 2 diabetes mellitus and comparative study of histologically proven nonalcoholic fatty liver diseases with or without type 2 diabetes mellitus. J Gastroenterol. 2013;48:515-25.

43. Seko Y, Sumida Y, Tanaka S, et al. Effect of sodium glucose cotransporter 2 inhibitor on liver function tests in Japanese patients with non-alcoholic fatty liver disease and type 2 diabetes mellitus. Hepatol Res. 2017;47:1072-8.

44. Komiya C, Tsuchiya K, Shiba K, et al. Ipragliflozin improves hepatic steatosis in obese mice and liver dysfunction in type 2 diabetic patients irrespective of body weight reduction. PLoS ONE. 2016;11:e0151511

45. Takase T, Nakamura A, Miyoshi H, Yamamoto C, Atsumi T. Amelioration of fatty liver index in patients with type 2 diabetes on ipragliflozin: an association with glucose-lowering effects. Endocr J. 2017;64:363-7

46. Takeda A, Irahara A, Nakano A, et al. The improvement of the hepatic histological findings in a patient with non-alcoholic steatohepatitis with type 2 diabetes after the administration of the sodium-glucose cotransporter 2 inhibitor ipragliflozin. Intern Med. 2017;56:2739-44

47. Akuta N, Watanabe C, Kawamura Y, et al. Effects of a sodiumglucose cotransporter 2 inhibitor in nonalcoholic fatty liver disease complicated by diabetes mellitus: preliminary prospective study based on serial liver biopsies. Hepatol Commun. 2017;1:46-52.

48. Shibuya T, Fushimi N, Kawai M, et al. Luseogliflozin improves liver fat deposition compared to metformin in type 2 diabetes patients with non-alcoholic fatty liver disease: a prospective randomized controlled pilot study. Diabetes Obes Metab (In press).

49. Ito D, Shimizu S, Inoue K, et al. Comparison of ipragliflozin and pioglitazone effects on nonalcoholic fatty liver disease in patients with type 2 diabetes: a randomized, 24-week, openlabel, active-controlled trial. Diabetes Care. 2017;40:1364-72

50. Yoneda M, Fujita K, Nozaki Y, et al. Efficacy of ezetimibe for the treatment of non-alcoholic steatohepatitis: an open-label, pilot study. Hepatol Res. 2010;40:566-73.

51. Park H, Shima T, Yamaguchi K, et al. Efficacy of long-term ezetimibe therapy in patients with nonalcoholic fatty liver disease. J Gastroenterol. 2011;46:101-7.

52. Loomba R, Sirlin CB, Ang B, et al; San Diego Integrated NAFLD Research Consortium (SINC). Ezetimibe for the treatment of nonalcoholic steatohepatitis: assessment by novel magnetic resonance imaging and magnetic resonance elastography in a randomized trial (MOZART trial). Hepatology. 2015;61:1239-50.

53. Nakade Y, Murotani K, Inoue T, et al. Ezetimibe for the treatment of non-alcoholic fatty liver disease: a meta-analysis. Hepatol Res (In press).

54. Sanyal AJ, Abdelmalek MF, Suzuki A, et al; EPE-A Study Group. No significant effects of ethyl-eicosapentanoic acid on histologic features of nonalcoholic steatohepatitis in a phase 2 trial. Gastroenterology. 2014;147:377-84.

55. Scorletti E, Bhatia L, McCormick KG, et al; WELCOME Study. Effects of purified eicosapentaenoic and docosahexaenoic acids in nonalcoholic fatty liver disease: results from the Welcome* study. Hepatology. 2014;60:1211-21.

56. Singh S, Singh PP, Singh AG, et al. Statins are associated with a reduced risk of hepatocellular cancer: a systematic review and meta-analysis. Gastroenterology. 2013;144:323-32.

57. Ishibashi S, Yamashita S, Arai H, et al; K-877-04 Study Group. Effects of K-877, a novel selective PPAR $\alpha$ modulator (SPPARM $\alpha$ ), in dyslipidaemic patients: a randomized, double blind, active- and placebo-controlled, phase 2 trial. Atherosclerosis. 2016;249:36-43.

58. Honda Y, Kessoku T, Ogawa Y, et al. Pemafibrate, a novel selective peroxisome proliferator-activated receptor alpha modulator, improves the pathogenesis in a rodent model of nonalcoholic steatohepatitis. Sci Rep. 2017;7:42477

59. Gilat T, Leikin-Frenkel A, Goldiner L, et al. Arachidyl amido cholanoic acid (Aramchol) is a cholesterol solubilizer and prevents the formation of cholesterol gallstones in inbred mice. Lipids. 2001;36:1135-40.

60. Safadi R, Konikoff FM, Mahamid M, et al; FLORA Group. The fatty acid-bile acid conjugate Aramchol reduces liver fat content in patients with nonalcoholic fatty liver disease. Clin Gastroenterol Hepatol. 2014;12:2085-91.

61. Foster DW. Malonyl-CoA: the regulator of fatty acid synthesis and oxidation. J Clin Invest. 2012;122:1958-9.

62. Yokohama S, Yoneda M, Haneda M, et al. Therapeutic efficacy of an angiotensin II receptor antagonist in patients with nonalcoholic steatohepatitis. Hepatology. 2004;40:1222-5.

63. Georgescu EF, Ionescu R, Niculescu M, et al. Angiotensin-receptor blockers as therapy for mild-to-moderate hypertension- 
associated non-alcoholic steatohepatitis. World J Gastroenterol. 2009; 15:942-54.

64. McPherson S, Wilkinson N, Tiniakos D, et al. A randomised controlled trial of losartan as an anti-fibrotic agent in non-alcoholic steatohepatitis. PLoS ONE. 2017;12(4):e0175717.

65. Neuschwander-Tetri BA, Loomba R, Sanyal AJ, et al; NASH Clinical Research Network. Farnesoid $\times$ nuclear receptor ligand obeticholic acid for non-cirrhotic, non-alcoholic steatohepatitis (FLINT): a multicentre, randomised, placebo-controlled trial. Lancet. 2015;385(9972):956-65.

66. McMahan RH, Wang XX, Cheng LL, et al. Bile acid receptor activation modulates hepatic monocyte activity and improves nonalcoholic fatty liver disease. $J$ Biol Chem. 2013;288:11761-70.

67. Vatner DF, Weismann D, Beddow SA, et al. Thyroid hormone receptor- $\beta$ agonists prevent hepatic steatosis in fat-fed rats but impair insulin sensitivity via discrete pathways. Am J Physiol Endocrinol Metab. 2013;305:E89-100. Lipids. 2001;36:1135-40.

68. Zein CO, Yerian LM, Gogate P, et al. Pentoxifylline improves nonalcoholic steatohepatitis: a randomized placebo-controlled trial. Hepatology. 2011;54:1610-9.

69. Du J, Ma YY, Yu CH, et al. Effects of pentoxifylline on nonalcoholic fatty liver disease: a meta-analysis. World J Gastroenterol. 2014;20:569-77.

70. Brenner C, Galluzzi L, Kepp O, Kroemer G. Decoding cell death signals in liver inflammation. J Hepatol. 2013;59:583-94.

71. Loomba R, Lawitz E, Mantry PS, et al. The ASK1 inhibitor selonsertib in patients with nonalcoholic steatohepatitis: a randomized, phase 2 trial. Hepatology (In press).

72. Barreyro FJ, Holod S, Finocchietto PV, et al. The pan-caspase inhibitor Emricasan (IDN-6556) decreases liver injury and fibrosis in a murine model of nonalcoholic steatohepatitis. Liver Int. 2015;35:953-66.

73. Weston CJ, Shepherd EL, Claridge LC, et al. Vascular adhesion protein-1 promotes liver inflammation and drives hepatic fibrosis. J Clin Invest. 2015;125:501-20.

74. Mizrahi M, Shabat Y, Ben Ya'acov A, et al. Alleviation of insulin resistance and liver damage by oral administration of Imm124-E is mediated by increased Tregs and associated with increased serum GLP-1 and adiponectin: results of a phase I/II clinical trial in NASH. J Inflamm Res. 2012;5:141-50.

75. Rivera CA, Adegboyega P, van Rooijen N, et al. Toll-like receptor-4 signaling and Kupffer cells play pivotal roles in the pathogenesis of non-alcoholic steatohepatitis. J Hepatol. 2007;47:571-9.

76. Hagström H, Nasr P, Ekstedt M, et al. Fibrosis stage but not NASH predicts mortality and time to development of severe liver disease in biopsy-proven NAFLD. J Hepatol. 2017;67:1265-73

77. Di Prospero NA, Artis E, Andrade-Gordon P, et al. CCR2 antagonism in patients with type 2 diabetes mellitus: a randomized, placebo-controlled study. Diabetes Obes Metab. 2014;16:1055-64.

78. Marra F, Tacke F. Roles for chemokines in liver disease. Gastroenterology. 2014;147(3):577-94.

79. Friedman SL, Ratziu V, Harrison SA, et al. A randomized, placebo-controlled trial of cenicriviroc for treatment of nonalcoholic steatohepatitis with fibrosis. Hepatology (In press).

80. Moon HJ, Finney J, Ronnebaum T, et al. Human lysyl oxidaselike 2. Bioorg Chem. 2014;57:231-41.

81. Henderson NC, Sethi T. The regulation of inflammation by galectin-3. Immunol Rev. 2009;230:160-71.

82. Harrison SA, Marri SR, Chalasani N, et al. Randomised clinical study: GR-MD-02, a galectin-3 inhibitor, vs. placebo in patients having non-alcoholic steatohepatitis with advanced fibrosis. Aliment Pharmacol Ther. 2016;44:1183.

83. Itoh N, Nakayama Y, Konishi M. Roles of FGFs as paracrine or endocrine signals in liver development, health, and disease. Front Cell Dev Biol. 2016;4:30.

84. Gaich G, Chien JY, Fu H, et al. The effects of LY2405319, an FGF21 analog, in obese human subjects with type 2 diabetes. Cell Metab. 2013;18:333-40.

85. Leeming D, et al. Estimation of serum "true collagen type III formation" (Pro-C3) levels as a marker of non-alcoholic steatohepatitis in a prospective cohort. J Hepatol. 2017;66(Suppl1):S154.

86. Reilly SM, Chiang SH, Decker SJ, et al. An inhibitor of the protein kinases TBK1 and IKK- $\varepsilon$ improves obesity-related metabolic dysfunctions in mice. Nat Med. 2013;19:313-21.

87. Costabel U, Albera C, Lancaster LH, Lin CY, Hormel P, Hulter HN, Noble PW. An open-label study of the long-term safety of pirfenidone in patients with idiopathic pulmonary fibrosis (RECAP). Respiration. 2017;94:408-15

88. Komiya C, Tanaka M, Tsuchiya K, et al. Antifibrotic effect of pirfenidone in a mouse model of human nonalcoholic steatohepatitis. Sci Rep. 2017;7:44754.

89. Hoofnagle JH, Van Natta ML, Kleiner DE, et al; Non-alcoholic Steatohepatitis Clinical Research Network (NASH CRN). Vitamin $\mathrm{E}$ and changes in serum alanine aminotransferase levels in patients with non-alcoholic steatohepatitis. Aliment Pharmacol Ther. 2013;38:134-43.

90. Seko Y, Sumida Y, Tanaka S, et al. Serum alanine aminotransferase predicts the histological course of non-alcoholic steatohepatitis in Japanese patients. Hepatol Res. 2015;45:E53-61.

91. Vilar-Gomez E, Yasells-Garcia A, Martinez-Perez Y, et al. Development and validation of a noninvasive prediction model for nonalcoholic steatohepatitis resolution after lifestyle intervention. Hepatology. 2016;63:1875-87.

92. Hamaguchi E, Takamura T, Sakurai M, et al. Histological course of nonalcoholic fatty liver disease in Japanese patients: tight glycemic control, rather than weight reduction, ameliorates liver fibrosis. Diabetes Care. 2010;33:284-6.

93. Shah AG, Lydecker A, Murray K, et al; Nash Clinical Research Network. Comparison of noninvasive markers of fibrosis in patients with nonalcoholic fatty liver disease. Clin Gastroenterol Hepatol. 2009;7:1104-12.

94. Sumida Y, Yoneda M, Hyogo H, et al; Japan Study Group of Nonalcoholic Fatty Liver Disease (JSG-NAFLD). Validation of the FIB4 index in a Japanese nonalcoholic fatty liver disease population. BMC Gastroenterol. 2012;12:2.

95. Xiao G, Zhu S, Xiao X, et al. Comparison of laboratory tests, ultrasound, or MRE to detect fibrosis in patients with non-alcoholic fatty liver disease: a meta-analysis. Hepatology. 2017;66:1486-501

96. Yoneda M, Yoneda M, Mawatari H, et al. Noninvasive assessment of liver fibrosis by measurement of stiffness in patients with nonalcoholic fatty liver disease (NAFLD). Dig Liver Dis. 2008;40:371-8.

97. Seki K, Shima T, Oya H, et al. Assessment of transient elastography in Japanese patients with non-alcoholic fatty liver disease. Hepatol Res. 2017;47:882-9.

98. Okajima A, Sumida Y, Taketani H, et al. Liver stiffness measurement to platelet ratio index predicts the stage of liver fibrosis in non-alcoholic fatty liver disease. Hepatol Res. 2017;47:721-30.

99. Imajo K, Kessoku T, Honda Y, et al. Magnetic resonance imaging more accurately classifies steatosis and fibrosis in patients with nonalcoholic fatty liver disease than transient elastography. Gastroenterology. 2016;150:626-37. 
100. Kwok R, Choi KC, Wong GL, et al. Screening diabetic patients for non-alcoholic fatty liver disease with controlled attenuation parameter and liver stiffness measurements: a prospective cohort study. Gut. 2016;65:1359-68.

101. Roulot D, Roudot-Thoraval F, NKontchou G, Kouacou N, Costes JL, Elourimi G, Le Clesiau H, Ziol M, Beaugrand M. Concomitant screening for liver fibrosis and steatosis in French type 2 diabetic patients using Fibroscan. Liver Int. 2017;37:1897-906

102. Koehler EM, Plompen EP, Schouten JN, et al. Presence of diabetes mellitus and steatosis is associated with liver stiffness in a general population: the Rotterdam study. Hepatology. 2016;63:138-47.

103. Doycheva I, Cui J, Nguyen P, et al. Non-invasive screening of diabetics in primary care for NAFLD and advanced fibrosis by MRI and MRE. Aliment Pharmacol Ther. 2016;43:83-95.
104. Nakahara T, Hyogo H, Yoneda M, et al; Japan Study Group of Nonalcoholic Fatty Liver Disease. Type 2 diabetes mellitus is associated with the fibrosis severity in patients with nonalcoholic fatty liver disease in a large retrospective cohort of Japanese patients. J Gastroenterol. 2014;49:1477-84.

105. Ong JP, Pitts A, Younossi ZM. Increased overall mortality and liver-related mortality in non-alcoholic fatty liver disease. J Hepatol. 2008;49:608-12.

106. Oza N, Eguchi Y, Mizuta T, et al. A pilot trial of body weight reduction for nonalcoholic fatty liver disease with a home-based lifestyle modification intervention delivered in collaboration with interdisciplinary medical staff. $\mathrm{J}$ Gastroenterol. 2009;44:1203-8.

107. Younossi ZM, Blissett D, Blissett R, et al. The economic and clinical burden of nonalcoholic fatty liver disease in the United States and Europe. Hepatology. 2016;64:1577-86. 